\title{
Rectal spirochaetosis in homosexual men: the association with sexual practices, HIV infection and enteric flora
}

\author{
C L H Law, J M Grierson, S M B Stevens
}

\begin{abstract}
Objective-To determine the prevalence of rectal spirochaetosis in homosexual men attending a sexually transmissible diseases clinic and investigate the association between their presence and sexual practices, HIV infection and enteric flora.

Design-The study included 144 male homosexual subjects who each completed a questionnaire, underwent physical examination, proctoscopy and investigations for STD and HIV screening, rectal biopsies and collection of faecal samples. Setting-The Sexual Health Centre, Sydney Hospital, Sydney, Australia.

Results-Spirochaetes were detected in $39 \%$ of the rectal biopsies, using histological criteria. Logistic regression analysis showed that rectal spirochaetosis was significantly associated with: oral-anal contact. ( $P<0.05$, OR 3.45, 95\% CI 1.48-8.05); detection of 3-5 different nonpathogenic protozoa in faeces $(P<0.01$, OR 11.68, 95\% C.I. 2.33-58) and a positive HIV antibody test $(P<0.01)$ OR 4.48, 95\% C.I. 1·28-15·72).

Conclusions-These findings indicate that rectal spirochaetosis is relatively common in homosexual men. The association with non-pathogenic protozoa is most likely attributable to the common mode of transmission viz oral-anal contact. However it is difficult to determine whether the association with HIV infection is cause or effect because of the limitations in the study design. Further information is required to determine the clinical significance of infection with these organisms.
\end{abstract}

(Genitourin Med 1994;70:6-29)

Sexual Health Centre, Sydney Hospital, Sydney, Australia C L H Law

Department of Anatomical Pathology, ICPMR Westmead Hospital, Sydney, Australia J M Grierson

$S$ M B Stevens

Address correspondence to Dr Carmella Law, Sexual Health Clinic, St George Hospital, 1st floor, 36 Belgrave Street, Kogarah, N.S.W. 2217, Australia

Accepted for publication 13 October 1993

\section{Introduction}

Spirochaetes in stool and rectal mucosa have been recognised since 1884, and in the last 10 years it has become apparent that they are more common in homosexual men ${ }^{12}$ (30-36\%) than, for instance, in unselected patients undergoing sigmoidoscopy or surgery $^{34}(1 \cdot 9-6 \cdot 9 \%)$. However their clinical significance is controversial, even in homosexual men with AIDS and severe immunodeficiency. The aim of this study was to determine the prevalence of rectal spirochaetosis in a group of mainly healthy homosexual men and to attempt to correlate their presence with that of other intestinal pathogens, sexual practices and HIV infection.

\section{Patients and methods}

Between 1985-1990, our group was involved in a study of STD and bowel conditions in homosexual men attending the Sydney Sexual Health Centre; the project was approved by the Ethics Committee of Sydney Hospital. These were healthy unselected homosexual men who presented to one of us (CL) and enrolled in the study after giving informed consent. All subjects completed a self-administered questionnaire to record past medical and STD history, history of gastrointestinal symptoms and details of sexual practices. They also underwent a full physical examination by the same investigator (CL) at every visit to ensure uniformity. On the first visit all subjects were offered routine assessment for STD and tests of immune function: Human immunodeficiency virus (HIV) I antibody by ELISA (Abbott Recombinant HIVEIA, Abbott Laboratories, North Chicago, USA and Wellcozyme anti-HIV III EIA, Wellcome Diagnostics, Beckenham, UK). Positive antibody tests were confirmed by western blot (DuPont Australia Ltd). Serological tests for treponemal infections namely: Veneral Diseases Reference Laboratory (VDRL) and Treponema pallidum haemaglutination (TPHA) tests;

Cultures for Neisseria gonorrhoeae and Chlamydia trachomatis from the rectum, urethral meatus and pharynx; and cultures for herpes simplex virus from the rectum only; Serum immunoglobulin levels and quantitation of peripheral blood T-lymphocytes subsets.

\section{Rectal biopsies}

Proctoscopy was performed using disposable sigmoidoscopes and a Heine light source. Three rectal biopsies were taken under direct vision, with flexible colonoscopy biopsy forceps, at 15, 10 and $5 \mathrm{~cm}$ from the anal verge. The two lower biopsies were placed into Millonig's buffered formalin fixative for light microscopy. The $15 \mathrm{~cm}$ biopsy was placed in Karnovsky's fixative for electron microscopy.

The formalin fixed tissue was paraffin embedded, sectioned at three serial levels and stained with haematoxylin and eosin ( $\mathrm{H} \& \mathrm{E})$. Sections were examined for evidence of inflammatory or other pathological changes. ${ }^{5}$ A further section was stained by the Warthin-Starry silver stain for spirochaetes 
adherent to the mucosa. For electron microscopy, the Karnovsky's fixed biopsy was routinely processed for tissue samples, without uranyl acetate. Grids were stained with uranyl acetate and lead citrate, and 2-4 areas were examined. If the presence of rectal spirochaetes was suspected on the histological sections, this was confirmed later by electron microscopy.

\section{Histopathological criteria for spirochaetosis}

Rectal spirochaetes was identified by the characteristic appearance on $\mathrm{H} \& \mathrm{E}$ staining of a haematoxyphilic haze along the luminal brush border surface of the mucosal epithelial cells, ${ }^{6}$ interrupted by goblet cells but sometimes extending some distance into crypts. The organisms detected in this way were also confirmed by positive silver staining on the Warthin-Starry stain.

\section{Electron microscopic criteria for diagnosis of spirochaetosis}

The spirochaetes occupy a zone approximately $0.2 \mu \mathrm{m}$ in diameter but since the whole length is not seen in section, measurement of length was not attempted. There is a trilaminar outer membrane and an inner cytoplasmic membrane. A periplasmic space contains flagella in which a substructure could not be discerned. Up to four flaggella were seen in optimal cuts. The cytoplasm contains ribosomes and thread-like nuclear material. The spirochaetes lie in regular arrangement parallel to the microvilli and at right angles to the surface of the mucosa. The regularity of the microvillous brush border is disorganised by the spirochaetes. The organisms invaginate the cell membrane but do not appear to invade the cytoplasm of the epithelial cells.

\section{Tests on faecal samples}

Each subject was asked to deliver a faecal sample on three separate days over a period of 1-2 weeks for the detection of potential enteric pathogens. All three specimens were examined for cysts, ova and parasites, specimens from the first two days were cultured for bacterial pathogens and the first only was examined for viruses. All laboratory procedures were performed in the Institute of Clinical Pathology and Medical Research (ICPMR) at Westmead Hospital, except for the parasitological investigations which were performed at the School of Public Health at the University of Sydney.

\section{Statistical Methods}

Pearson's chi square test with continuity correction and Fisher's exact test, where appropriate, were used to test the association between the variables collected and rectal spirochaetosis. The cutoffs for the continuous variables are as shown in the table. Non-pathogenic protozoans (NPP) were categorised by the number of different types of protozoans identified to simplify our investigation of their significance; therefore NPP were divided into three groups for both the chi square and logistic regression analysis. All variables found significant in the chi square analysis, and others that were considered clinically important, were entered into the logistic regression model to evaluate interactions while controlling for possible confounders.

\section{Results}

Rectal biopsies were performed in all 144 homosexual men and spirochaetes were detected in $56(39 \%)$. Of the 56 individuals, $13(9 \%)$ had occasional or patchy, $26(18 \%)$ moderate and $17(12 \%)$ heavy colonisation according to the histological appearance.

The mean age of the subjects was 34 years (range 21-68). The information on sexual practices was provided by only 124 subjects and was incomplete in some cases: $116 / 123$ (94\%) admitted to oral-genital contact, $66 / 124(53 \%)$ to oral-anal contact, $111 / 122$ $(91 \%)$ to receptive anal intercourse, $111 / 123$ $(90 \%)$ to insertive anal intercourse, and $9 / 122(7 \%)$ to "fisting" (insertion of the fist into the rectum). On the preliminary analysis, there was significant relationship between rectal spirochaetosis and oral-anal contact ( $p$ $<0.05$ ) alone but none of the other sexual practices, including receptive anal intercourse.

Immune function and HIV antibody tests were performed in most subjects and the results are summarized in the table. The prevalence of STD (reactive treponemal serology, genital warts, rectal herpes, rectal gonorrhoea and chlamydia infection) in this group has been reported elsewhere. ${ }^{5}$

Although the subjects did not present primarily because of gastrointestinal symptoms, on direct questioning and information obtained from questionnaires indicated that 34 of $138(25 \%)$ subjects for whom information was available, had had gastrointestinal symptoms for more than one week. Most reported mild symptoms such as loose stools, lower abdominal discomfort, bloated feeling, occasional nausea and/or flatulence. Rectal biopsies showed inflammatory changes consistent with proctitis in 35/143 (24\%) subjects. No association was noted between rectal spirochaetosis and gastrointestinal symptoms or proctitis (table).

The viral, bacterial and protozoal pathogens and non-pathogenic protozoa (NPP) identified in these subjects have been previously reported. ${ }^{57}$ The protozoa detected in faecal specimens included: Entamoeba histolytica, Giardia intestinalis, Dientamoeba fragilis and a variety of NPP. $E$ histolytica was detected in faecal specimens from 49/125 (39\%) of the study group and at least one NPP in $105 / 125(84 \%)$; similar proportions of subjects $(55 / 125(44 \%)$ and $50 / 125$ (40\%)) each carrying 1-2 and 3-5 NPP respectively. In the chi square analysis, we found that rectal spirochaetosis was associated with $E$ histolytica $(\mathrm{p}<0.05)$ and the presence of 3-5 NPP ( $p<0.01)$ (table).

HIV-antibody was detected in 61 of 138 (44\%) subjects tested of whom $53(87 \%)$ 
Table Association between sexual practices, bowel protozoa, gastro-intestinal symptoms, proctitis, $T$ cell subsets, immunoglobulins, HIV antibody result and rectal spirochaetosis.

\begin{tabular}{|c|c|c|c|c|}
\hline Variables: & $\begin{array}{l}\text { Total } \\
\text { No. with variable/No. } \\
\text { for whom data available }\end{array}$ & $\begin{array}{l}\text { Negative } \\
N(\%)\end{array}$ & $\begin{array}{l}\text { tosis } \\
\text { Positive } \\
N(\%)\end{array}$ & Odds Ratio \\
\hline $\begin{array}{l}\text { Number of years of homosexual activity }>11 \\
\text { Number of partners over the subjects' lifetime }>99 \\
\text { Oral-genital contact } \\
\text { Oral-anal contact } \\
\text { Receptive anal intercourse } \\
\text { Insertive anal intercourse } \\
\text { "Fisting" } \\
\text { Entamoeba histolytica }\end{array}$ & $\begin{array}{r}98 / 143 \\
99 / 144 \\
116 / 123 \\
66 / 124 \\
111 / 122 \\
111 / 123 \\
9 / 122 \\
49 / 125\end{array}$ & $\begin{array}{c}56 / 87(64 \cdot 4 \%) \\
55 / 88(62 \cdot 5 \%) \\
70 / 75(93 \cdot 3 \%) \\
33 / 76(43 \cdot 4 \%) \\
64 / 74(86 \cdot 5 \%) \\
67 / 75(89 \cdot 3 \%) \\
4 / 74(5 \cdot 4 \%) \\
23 / 77(29 \cdot 9 \%)\end{array}$ & $\begin{array}{r}42 / 56(75 \cdot 0 \%) \\
44 / 56(78 \cdot 6 \%) \\
46 / 48(95 \cdot 8 \%) \\
33 / 48(68 \cdot 7 \%) \\
47 / 48(97 \cdot 9 \%) \\
44 / 48(91 \cdot 7 \%) \\
5 / 48(10 \cdot 4 \%) \\
26 / 48(54 \cdot 2 \%)\end{array}$ & $\begin{array}{l}1 \cdot 68 \\
2 \cdot 20 \\
1 \cdot 64 \\
2 \cdot 87^{\star} \\
7 \cdot 34 \\
1 \cdot 31 \\
2 \cdot 03 \\
2 \cdot 77^{\star}\end{array}$ \\
\hline \multicolumn{5}{|l|}{ Non-pathogenic protozoans: } \\
\hline $\begin{array}{l}1-2 \text { NPP vs NEG } \\
3-5 \text { NPP vs NEG }\end{array}$ & $\begin{array}{l}55 / 125 \\
50 / 125\end{array}$ & $\begin{array}{l}36 / 77(46 \cdot 8 \%) \\
23 / 77(29 \cdot 9 \%)\end{array}$ & $\begin{array}{l}19 / 48(39 \cdot 6 \%) \\
27 / 48(56: 3 \%)\end{array}$ & $\begin{array}{l}4 \cdot 75 \\
9 \cdot 66 t\end{array}$ \\
\hline Gastro-intestinal symptoms & $34 / 138$ & $23 / 83(27 \cdot 7 \%)$ & $11 / 55(20.0 \%)$ & 0.65 \\
\hline Proctitis & $35 / 143$ & $22 / 87(25 \cdot 3 \%)$ & $13 / 56(23 \cdot 2 \%)$ & 0.91 \\
\hline HIV-antibody positive & $61 / 138$ & $34 / 84(40 \cdot 5 \%)$ & $27 / 54(50 \cdot 0 \%)$ & 1.47 \\
\hline $\begin{array}{l}\text { CD4 + Lymphocytes below lower limit NR }\left(360 / \mathrm{mm}^{3}\right) \\
\text { CD8 + Lymphocytes above upper limit NR }\left(660 / \mathrm{mm}^{3}\right)\end{array}$ & $\begin{array}{l}12 / 144 \\
83 / 144\end{array}$ & $\begin{array}{c}7 / 88(8 \cdot 0 \%) \\
52 / 88(59 \cdot 1 \%)\end{array}$ & $\begin{array}{l}5 / 56(8.9 \%) \\
31 / 56(55 \cdot 4 \%)\end{array}$ & $\begin{array}{l}0.94 \\
0.86\end{array}$ \\
\hline \multicolumn{5}{|l|}{ Immunoglobulins: } \\
\hline $\begin{array}{l}\text { Elevated IgG above upper limit NR }(193 \mathrm{IU} / \mathrm{ml}) \\
\text { Elevated IgM above upper limit NR }(240 \mathrm{IU} / \mathrm{ml})\end{array}$ & $\begin{array}{l}50 / 140 \\
52 / 140\end{array}$ & $\begin{array}{l}29 / 86(33 \cdot 7 \%) \\
35 / 86(40 \cdot 7 \%)\end{array}$ & $\begin{array}{l}21 / 54(38 \cdot 9 \%) \\
17 / 54(31 \cdot 5 \%)\end{array}$ & $\begin{array}{l}1 \cdot 25 \\
0 \cdot 71\end{array}$ \\
\hline Elevated IgA above upper limit NR (345IU $/ \mathrm{ml})$ & $13 / 140$ & $6 / 86(7 \cdot 0 \%)$ & $7 / 54(13 \cdot 0 \%)$ & 1.99 \\
\hline
\end{tabular}

${ }^{\star} \mathrm{p}<0.05$, tp $<0.01, \mathrm{NR}=$ normal range

were in CDC group II (asymptomatic) and 8 $(13 \%)$ in group III (persistent generalised lymphadenopathy). In the logistic regression analysis when rectal spirochaetosis was analysed together with other factors viz. sexual practices, immune function and proctitis, to examine which factors predicted HIVseropositivity, rectal spirochaetosis emerged as being significant ( $\mathrm{p}<0.01$, OR $4.48,95 \%$ CI 1.28-15.72) but the reverse was not true.

Again using logistic regression analysis but using rectal spirochaetosis as the dependent variable, the most significant findings were the relationship between rectal spirochaetosis and 3-5 different types of NPP and a history of oral-anal contact. The homosexual men who carried 3-5 NPP were more likely to have evidence of rectal spirochaetosis on their rectal biopsies than those who did not carry NPP $\quad(P<0.01$, OR 11.68, 95\% CI $2 \cdot 33-58 \cdot 57)$. As in the preliminary analysis, only the practice of oral-anal contact, and not the other sexual practices, was associated with an increased risk of spirochaetosis ( $p<0.05$, OR 3.45, 95\% CI 1.48-8.05).

\section{Discussion}

There is little information about the nature of intestinal spirochaetes and most published descriptions are based on light and electron microscopic appearances. These organisms constitute a heterogeneous group with those identified so far being provisionally allocated to several genera, including Serpulina (formerly Treponema), Brachyspira ${ }^{8}$ (new genus) and Anaerobiospirillum ${ }^{9}$ (a non-spirochaetal spiral bacterium). Only recently have suitable conditions for in vitro culture been established.

The significance of findings on electron microscopy that is, shortening and loss of microvilli in areas affected by spirochaetes and penetration into epithelial cells and adjacent tissues ${ }^{3810}$ is not fully understood. Although one spirochaete, Serpulina hyodysenteriae, is known to cause dysentery in swine and there have also been reports associating spirochaetes with gastrointestinal symptoms, ${ }^{11} 12$ in homosexual men, most investigators believe that they are non-pathogenic.

This study showed a high incidence of rectal spirochaetosis on histological examination of rectal biopsies from homosexual men, of whom $44 \%$ had HIV infection but were essentially healthy. There was a significant and independent association between the presence of spirochaetes and a history of oralanal contact, suggesting the transmission occurs by the faecal-oral route. This has not been postulated before despite the wellknown observations that rectal spirochaetes are at least five times more prevalent in homosexual men and that another organism accepted as being spread by a similar mechanism, $E$ histolytica, has also been detected more commonly in homosexual men ${ }^{7}$ than in the general population. This was supported by an association also with the presence of several different non-pathogenic protozoa in faeces, a finding usually taken to indicate faecal exposure. On the other hand rectal spirochaetosis was not associated with gastrointestinal symptoms or proctitis in this population.

Although we did not find a significant relationship between receptive anal intercourse and spirochaetosis, the possibility of this association has not been ruled out. This is because almost all subjects $(>90 \%)$ in this study reported oral-genital contact and anal intercourse, so that we cannot comment on whether these activities were indeed significant factors in the acquisition of rectal spirochaetes. In the homosexual men who practised receptive anal intercourse, some factor associated with this practice viz. localised trauma, deposition of semen or sexually transmissible organisms could lead to a suppression of the mucosal immunity and thereby increasing the likelihood of persistence or increase in dose of the rectal spirochaetes.

We also showed that homosexual men with rectal spirochaetosis were approximately four 
times more likely to be infected with HIV than those who had no evidence of spirochaetosis in their rectal biopsies. However, immune function, as determined by the measurement of peripheral $\mathrm{T}$-cell subsets and immunoglobulin levels, did not appear to be a significant factor in determining the presence of these organisms. A possible explanation for this may be that in this group of relatively healthy, asymptomatic homosexual men, peripheral $\mathrm{T}$ cell markers may not be sensitive indicators of mucosal immunity. Rectal spirochaetosis could be an early manifestation of altered bowel immunity, thereby explaining why the condition is more prevalent in the homosexual men. Some of the factors which may alter local immunity include: trauma with anal intercourse and use of pleasuring devices, deposition of semen with receptive anal intercourse and infections with organisms such as herpes simplex virus, human papilloma virus and Candida albicans. In view of the recent reports of the detection of HIV in colonic biopsy samples, ${ }^{13}$ there is a distinct possibility of the HIV exerting a direct immunosuppressive effect on the immuno-reactive cells in the rectal mucosa. This effect has not been quantified as yet. The main studies so far of the T-lymphocytes in the intestinal mucosa have been in symptomatic HIV positive patients ${ }^{14}$ where a significant decrease of total number of T-cells was noted but much remains to be learned about rectal mucosal immunity.

Interestingly, we noted an association between the carriage of $E$ histolytica and other protozoans and the detection of rectal spirochaetes. Little is known about the interrelationship between the various organisms in the so-called "gay bowel syndrome"15 and the situation is further complicated by opportunistic gastrointestinal infections in terminal AIDS patients. Gastrointestinal viruses, predominantly rotaviruses and adenoviruses, were detected more commonly in homosexual men with symptomatic HIV infection than in those who were HIV-seronegative ${ }^{16}$ but we did not find any association in this study between bacterial or viral enteric flora and rectal spirochaetes.

Further work is indicated to determine the impact of rectal spirochaetes on local mucosal immunity in the bowel. The presence of other enteric organisms like protozoans, bacterial or viral pathogens, in particular herpes simplex virus, can directly affect the immunity at this level but on the other hand, may reflect the outcome of alteration to the mucosal immunity of the homosexual host as a result of HIV infection.

This project was funded by the Sydney Hospital Foundation for Medical Research. We wish to thank the following: the late SRN. Geoff Holt, research assistant nurse; Dr M Qassim for data management; Mr Peter Thomson for supervision of the statistical analysis; Dr J Walker, parasitologist, formerly of the School of Public Health and Tropical Medicine, University of Sydney; staff of the Microbiology department, ICPMR, Westmead ICPMR, Westmead Hospital; Drs W Jones and S Kamath, ICPMR, Westmead Hospital; Prof G L Gilbert for supervision of the manuscript;
and all the participants in the study.

1 Surawicz C M, Roberts P L, Rompalo A M, et al. Intestinal spirochetosis in homosexual men. $\mathrm{Am} \mathcal{F} \mathrm{Med}$ 1987;82:587-92

2 McMillan A, Lee F D. Sigmoidoscopic and microscopic appearance of the rectal mucosa of homosexual men. Gut 1981;22:1035-41.

3 Takeuchi A, Jervis H R, Nakazawatt H, Robinson D M. Spiral shaped organisms on the surface colonic epithelium of the monkey and man. Am $\mathcal{f}$ Clin Nutr 1974; 27:1287-96.

4 Lee F D, Kraszewski A, Gordon J, et al. Intestinal spirochaetosis. Gut 1971;12:126,330.

5 Law C L H, Qassim M, Cunningham A L, et al. Nonspecific proctitis: association with human immunodeficiency virus infection in homosexual men. $\mathcal{F}$ Infect Dis 1992;165:150-4.

6 "Intestinal Spirochaetosis" in: Richard Whitehead Mucosal Biopsy of the Gastro-intestinal Tract 3rd ed. 1985:295-7.

7 Law C L H, Walker J, Qassim M. Factors associated with the detection of Entamoeba histolytica in homosexual men. Int $₹$ STD \& AIDS 1991;2:346-50.

8 Hovind-Hougen $K$, Anderson A B, Neilsen $R H$. Intestinal spirochaetosis: morphological characterization Intestinaliven of the and cultivation of the spirochaete Brachyspira alborgi

gen nov. $\mathcal{F}$ Clin Microbiol 1982,16:1127-36.

Malnick H, Mem T, Lotay $\mathrm{H}$, et al. Anaerobiospiriullum species isolated from humans with diarrhoea. $\mathcal{F}$ Clin Pathol 1983;36:1097-1101.

10 Cooper C, Cotton D W K, Hudson M J, et al. Rectal spirochaetosis in homosexual men: characterisation of the organism and pathophysiology. Genitourin Med 1986;62:47-52.

11 Kaplan L, Takeuchi A. Purulent rectal discharge associated with a non treponemal spirochete. $¥ A M A 1979$; 241:52-3.

12 Gad A, Willen R, Furgard $\mathrm{K}$, et al. Intestinal spirochaetosis as a cause of long standing diarrhoea. Uppsala $\mathcal{F} \mathrm{Med}$ Sci 1977;82:49-54

13 Nelson J A, Wiley C A, Reynolds-Kohler C, et al. Human immunodeficiency virus in bowel epithelium from patients with gastrointestinal symptoms. Lancet 1988;1: patients

14 Rodgers V D, Fassett R, Kagnoff M F. Abnormalities in intestinal mucosal $\mathrm{T}$-cells in homosexual populations including those with the lymphadenopathy syndrome and AIDS. Gastroenterology 1986;90:552-8.

15 Sohn N, Robilotti J C. The gay bowel syndrome. A review of colonic and rectal conditions in 200 male homosexual. $A m \mathcal{F}$ Gastroenterol 1977;67:478-84.

16 Cunningham A L, Grohman G S, Harkness J, et al. Gastrointestinal viral infections in homosexual men who were symptomatic and seropositive for Immunodeficiency virus. $\mathcal{F}$ Infect Dis 1988;158:386-391. 